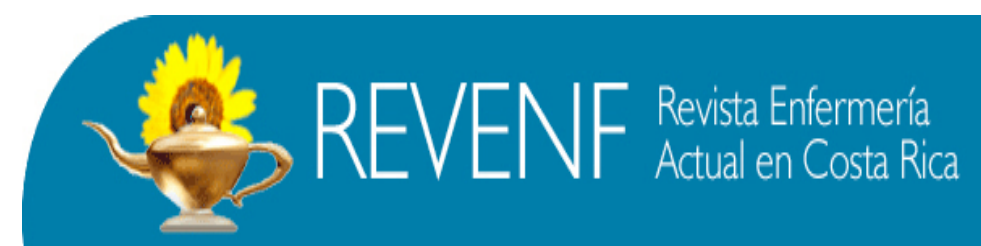

Revista Semestral Número 17 Octubre 2009-Marzo2010

ISSN 1409-4568

\title{
EL CAMPAMENTO COMO ESTRATEGIA METODOLÓGICA PARA FORTALECER LAS HABILIDADES PARA LA VIDA DE LOS JÓVENES ESTUDIANTES DE ENFERMERÍA ${ }^{1}$
}

\section{COMO CITAR ESTE ARTÍCULO}

Murillo Castro, Ligia; Muñoz Rojas, Derby; Elizondo Salazar, Humberto. El campamento como estrategia metodológica para fortalecer las habilidades para la vida de los jóvenes estudiantes de Enfermería. Rev. Enfermería Actual en Costa Rica [en línea].2009, No.17 [citado (fecha)]. Disponible World Wide Web: <http://www.revenf.ucr.ac.cr/factores.pdf> ISSN 1409-4568

\author{
Ligia Murillo Castro ${ }^{2}$ \\ Derby Muñoz Rojas ${ }^{3}$ \\ Humberto Elizondo Salazar ${ }^{4}$
}

\section{RESUMEN}

El campamento como estrategia metodológica para fortalecer las habilidades para la vida de los jóvenes estudiantes de Enfermería, consistió en el desarrollo de un encuentro, con el propósito fundamental de promover el desarrollo humano de los jóvenes que están cursando el tercer año de la carrera de licenciatura en Enfermería de la Universidad de Costa Rica. El estudio es cualitativo-participativo, con énfasis en la metodología de habilidades para la vida, se trabajó con una población total de 100 estudiantes. El campamento se desarrollo concentrándose en aspectos prácticos de la vida diaria para la prevención de los problemas psicosociales de los jóvenes estudiantes, así como la adquisición de competencias y habilidades específicas a nivel físico, social, cognitivo, moral y vocacional. Los resultados evidenciaron según los relatos que manifestaron los estudiantes, un fortalecimiento en sí mismos, además de que permitió la cohesión grupal de estas generaciones. Como conclusión final, esta actividad demostró, un aumento significativo en las habilidades de los sujetos participantes para la expresión de sus sentimientos, como la identificación de los factores protectores y de riesgo, aspectos con lo que cuentan para enfrentar los desafíos de la vida cotidiana como personas y como profesionales.

Palabras Claves: campamento, habilidades para la vida, inteligencia emocional, programación neurolingüística.

\footnotetext{
${ }^{1}$ Fecha de recepción: Marzo 2009

Fecha de aceptación: Junio 2009

${ }^{2}$ Master en Administración Pública, Licenciada en Enfermería. Profesora Escuela de Enfermería, Universidad de Costa Rica. Correo electrónico: ligia.murillo@ucr.ac.cr

${ }^{3}$ Licenciado en Enfermería. Profesor Escuela de Enfermería, Universidad de Costa Rica. Correo electrónico: derby.muñoz@ucr.ac.cr

${ }^{4}$ Master en Psicopedagogía. Instituto Costarricense sobre Drogas. San José, Costa Rica. Correo electrónico:helizondo@icd.go.cr
} 


\begin{abstract}
The camp like methodological strategy to strengthen the skills for the life of the young students of nursing consisted of the development of a meeting, with the fundamental intention of promoting the human development of the young students that they are dealing the third year of the career of licentiate in nursing in the University of Costa Rica. The study was of type of qualitative - participative research, emphatically in the methodology of skills for the life, in the investigation participated a total population of 100 students. The camp was develop in practical aspects of the daily life for the prevention of the psychosocial problems of the young students, as well as the acquisition of competitions and specific skills to physical, social, cognitive, moral and vocational level. The results demonstrated according to the statements given by the students, a strengthening of them selves, besides the study allowed the group cohesion of these generations. Final conclusion, this activity demonstrated, a significant increase in the skills of the fastened participants for the expression of feelings, as the identification of the protective and risk factors, which they have to face the challenges of the daily life as persons and professionals.
\end{abstract}

Key Words: camp, skills for the life, emotional intelligence, neurolinguistic programation

\section{INTRODUCCIÓN.}

La experiencia de la institución en la enseñanza de la educación superior en Enfermería, ha permitido el desarrollo de un plan de estudios basado en la promoción de la salud, prevención, curación y rehabilitación de la enfermedad, acentuando en el ciclo de vida del ser humano (niño, adolescente, adulto y adulto mayor ) específicamente en el programa de la licenciatura en enfermería, en el curso de tercer año de la carrera, en la particularidad de Módulo de Intervención de Enfermería en la Adolescencia. En el campo de la formación académica los estudiantes y docentes desarrollan actividades teóricos prácticas, concernientes al cuidado de los problemas psicosociales de los adolescentes y jóvenes, basándose en el énfasis de la promoción de la salud y la prevención de la enfermedad de esta población concretamente, experiencia desarrollada en los colegios de secundaria públicos y privados del área metropolitana, en los equipos básicos de atención integral (EBAIS) con la atención del embarazo en la adolescencia; en las Organizaciones gubernamentales y no gubernamentales (ONGs) del país con el abordaje del consumo de drogas.

El incursionar en este ámbito permitió evaluar y replantear para el grupo de docentes la importancia de fortalecer y empoderar al estudiante de Enfermería, porque también son personas expuestas a los mismos problemas de la adolescencia como grupo social. Se determinó que los estudiantes de Enfermería necesitan el fortalecimiento suficiente de sus herramientas personales, reflexionando acerca de sus capacidades, promoviendo los estilos de vida saludables para la toma de decisiones y el desarrollo de una autonomía individual, con la finalidad de facilitar el acercamiento y la comunicación con las poblaciones de adolescentes, así como la intervención de 
Enfermería hacia los distintos riesgos a las que se enfrentan actualmente los jóvenes.

\section{Punto de partida.}

A partir del año 2000, con la experiencia del desarrollo del Módulo de intervención de Enfermería en la Adolescencia, ubicado en el tercer año de la carrera de Enfermería, se inició la estrategia de abordaje en la modalidad de campamento, dirigido a los jóvenes estudiantes de Enfermería. El espacio físico seleccionado estuvo proyectado fuera del aula y del campus universitario, para lo cual ha sido destinado la Laguna de Fraijanes en Poas de la provincia de Alajuela, un bosque con senderos y cabañas donde la naturaleza y el contacto con ella forma parte del medio natural donde se desarrollan las acciones propias de la experiencia de aprendizaje. La finalidad de esta actividad es la de mejorar la condición humana de los jóvenes protagonistas para el fortalecimiento de la autoestima, la confianza en sí mismo (a), el facilitarles su inserción y la interacción en el ámbito de la experiencia cognitiva con los grupos de adolescentes, además de aprender a diseñar programas de intervención dirigidos a estas poblaciones. $\mathrm{La}$ enseñanza de estas destrezas psicosociales requieren de la discusión, el análisis, así como también de la reflexión de los valores que son parte del comportamiento de la niñez, adolescencia y juventud en la época actual.

La experiencia del campamento permitió plantear los siguientes objetivos en los estudiantes de Enfermería:

1. Promover el desarrollo humano.

2. Fomentar el crecimiento personal y los procesos de cambio.
3. Implementar herramientas de comunicación asertiva consigo mismo y con los demás.

4. Construir estrategias pedagógicas para el abordaje de grupos de adolescentes y jóvenes.

5. Fortalecer los factores protectores del adolescente.

6. Impulsar la criticidad, solidaridad, autonomía, el respeto y la tolerancia.

\section{Desarrollo de la temática.}

Tradicionalmente en los sistemas de salud de los países, la mayoría de los profesionales de la salud y las ciencias sociales han sido formados con el Modelo del Riesgo el cual Wolin y Wolin, 1993, tomado de Badilla (1999) mencionan que la familia con problemas produce daño y patología. Este modelo es importante de mencionarlo cuando se aborda poblaciones de niños, adolescentes y jóvenes ya que la familia como grupo social primario, tiene dificultades para enfrentar la crisis en alguno de sus miembros y como consecuencia se produce desintegración. Ante estas situaciones críticas, la familia declina ante el daño, es como un sufrimiento irremediable, que afecta a cada uno de sus miembros. En esta situación particular, se recomienda el tratar al niño, adolescente en el sistema de salud, como grupo familiar, que puede afectarse ante las dificultades que viven cotidianamente, situación que es una realidad ante los problemas que enfrenta la sociedad del presente en el mundo globalizado.

Sin embargo, otro abordaje de los adolescentes en grupo que se propone para la solución de sus problemas, es el modelo de la programación neurolingüística, de acuerdo a Albala, 
Salinas, y Vio (2004), éste fue destacado en los estudios de Richard Bandler y John Grinder (1972) en donde se matizan los patrones del lenguaje conocido como programación neurolingüística, también llamados metamodelos. En este proceso propuesto por estos autores representa una herramienta terapéutica, que explica cómo ayudar a la personas a realizar cambios en su vida diaria.

El autor Dilts (2004) propone la programación neurolingüística, (PNL) como un enfoque para el abordaje con adolescentes y jóvenes, el cual consiste en el término de niveles lógicos que fue adaptado del trabajo de Tim Bates en (1980) y se refiere a la jerarquía de nivel de proceso dentro del individuo o grupo, el cual pueden ser cambios a través del transcurso de la vida en niños $y$ adolescentes. La función de cada nivel consiste en sintetizar, organizar y dirigir las interacciones en un nivel inmediatamente inferior. Por lo que pasar a un nivel superior "irradiará" necesariamente hacia abajo, precipitándole cambio hacia los niveles superiores. Estos niveles incluyen (de superior a inferior) delimitados en la persona del adolescente en cambios que contienen los aspectos tales como: identidad, valores, creencias, capacidades, comportamiento y medio o entorno. Hay un sexto nivel, que el autor denomina espiritual y que puede ser definido como una especie de "campo de relaciones" que abarca múltiples identidades y da pie al sentimiento de ser miembro de un sistema mayor, más allá de la identidad individual. Muy ligado a este enfoque de la programación neurolingüística, se encuentra también la conformación de las capacidades individuales y con el entorno, planteadas por Cooper et al
(1998) llamado la inteligencia emocional, la cual es definida como Cooper y Sawaf (1998):

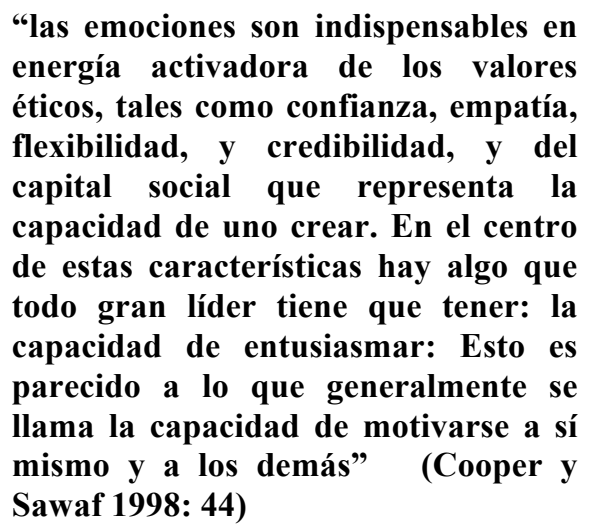

Este planteamiento de la inteligencia emocional es un modelo alternativo, para que los profesionales en la salud y de otras disciplinas de las ciencias sociales, puedan utilizarlo como un instrumento que le permita hacer la intervención de los adolescentes y jóvenes, en las diversas etapas del desarrollo en que se encuentran y las diferentes formas de comportamientos que asumen habitualmente.

Ante los diversos problemas psicosociales ya conocidos, que afectan a la población de adolescentes, como: delincuencia, narcotráfico, pandillas y promiscuidad sexual en los diversos países, el autor Mantilla (2001) a través del Ministerio de salud en Bogotá Colombia elaboró los instrumentos didácticos para la educación formal en el enfoque de las habilidades para la vida tales como: expresión de sentimientos, conflictos y la asertividad, estos antecedentes representan la base para el diseño la herramienta didáctica de la propuesta pedagógica para la promoción del desarrollo humano y la prevención de los problemas psicosociales de los jóvenes en la actualidad. 
Los enfoques descritos anteriormente como: la programación neurolingüística, la inteligencia emocional y las habilidades para la vida, han permitido el fundamento de las actividades de esta investigación participativa, dirigida a los estudiantes de Enfermería, para fortalecerlos a través del empoderamiento, ante los desafíos de la vida cotidiana, tal y como se evidencia en la figura 1 que a continuación se presenta:

\section{Figura 1}

Modelo de abordaje metodológico

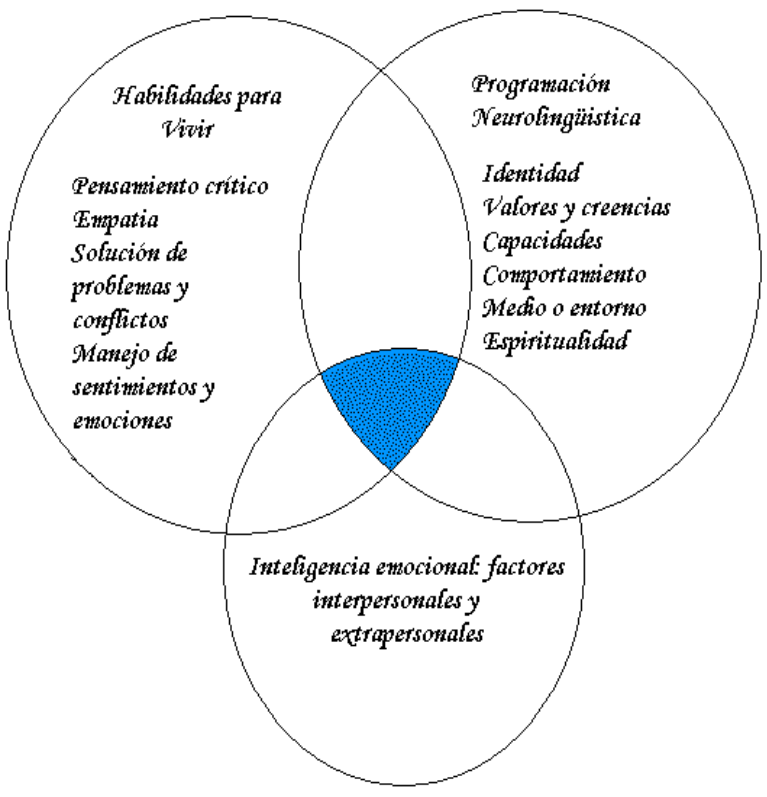

Figura 1. Traslape común entre las habilidades para la vida, y el enfoque de la programación neurolinguistica y la inteligencia emocional.

Este modelo metodológico, le permite al docente desarrollar diversos niveles de aprendizaje, en un proceso dinámico y meramente constructivista para el desarrollo y el fortalecimiento del adolescente y joven, asimismo es un enfoque de intervención para abordar los problemas a los que se enfrentan los jóvenes en la actualidad.

\section{CONSIDERACIONES ETICAS.}

En cuanto a las consideraciones éticas de la investigación, el estudiante de enfermería participó en forma voluntaria, para lo cual se le guardo absoluta confidencialidad, no se utilizaron los nombres que pudieran identificar a las personas participantes en el proceso investigativo.

\section{MATERIALES Y MÉTODOS.}

El diseño investigativo fue planteado desde el paradigma de la investigación cualitativa, el cual según Martínez (2006) este enfoque trata de identificar la naturaleza profunda de las realidades, su estructura dinámica, aquella que da razón plena de su comportamiento y manifestaciones en un todo integrado. Se trabajó además con la modalidad de investigación participativa donde los involucrados en este tipo de investigación figuran en el proceso en forma activa, se propone que se empoderen del conocimiento y es descrita por Vejarano (1983) como:

"Una actividad integrada que
combina la investigación social, el
trabajo educativo y la acción. La
combinación de estos elementos
dentro del proceso interrelacionados
ofrece tanto a sus seguidores, como a
los comprometidos o iniciados, motivo
de estímulo y también de
dificultadad." (Vejarano, 1983:18)

La investigación participativa tiene como meta el mejoramiento del nivel de vida de la población y pretende lograr que los individuos involucrados en el proceso, estén concientes de sus propias habilidades y recursos para brindarles el apoyo necesario para su organización y movilización. (Vejarano, 1983) Desde esta perspectiva esta investigación dirigida hacia los estudiantes de 
enfermería, tiene como propósito que los y las jóvenes encuentren sus propias fortalezas a través de las diversas actividades participativas que se desarrollaron en el campamento.

Este proceso de aprendizaje se basó en otras estrategias didácticas en las que se encuentra la metodología habilidades para vivir, planteamiento que fomenta, los factores protectores y la resiliencia de los jóvenes, este enfoque promueve el desarrollo de las competencias atribuidas a las habilidades psisociales en la persona del estudiante de enfermería. A continuación se describen las principales premisas de la metodología habilidades para la vida, como propuesta pedagógica, según Mantilla (2000) propone que:

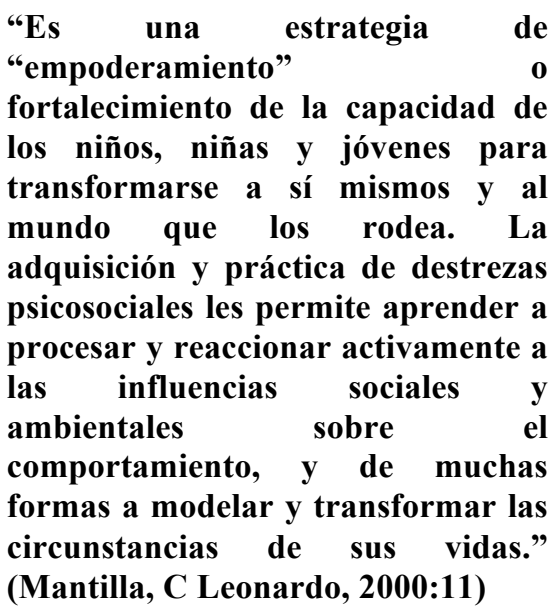

Esta metodología de Habilidades para Vivir permite según (Mantilla, 2000) la promoción de la salud, la prevención y el desarrollo humano, es una actividad concebida como el proceso de proporcionar a los pueblos los medios necesarios para que puedan mejorar su salud y ejercer un mayor control sobre ella, se fundamenta en un concepto de la salud mas integrador, como fuente de riqueza en la vida diaria, responsabilizando a todos los actores de la sociedad en este caso a las personas, la comunidad, las instituciones y los distintos sectores. Una de las cinco áreas de acción donde deben concentrarse los esfuerzos para la promoción de la salud, consiste precisamente en el desarrollo de aptitudes personales para tomar decisiones saludables y soportar presiones negativas del entorno, de manera que esta investigación participativa se apoyó en esta conceptualización.

La población estuvo caracterizada por la totalidad de los estudiantes de Enfermería que cursan el tercer año de la carrera y que están llevando el curso modulo de intervención de Enfermería en la adolescencia, la muestra estuvo representada por los estudiantes que voluntariamente decidieron participar en un campamento por año, el periodo comprendido entre el 2004 al 2006 que fue de un total de 100 estudiantes.

La investigación consistió en una primera etapa de coordinación, el docente organizador y los docentes del curso módulo de intervención de Enfermería en la adolescencia, realizan las diversas coordinaciones, efectuándose los enlaces: del lugar donde se realiza el campamento el cual es la Laguna de Fraijanes en la provincia de Alajuela, los recursos docentes y materiales se promovieron a través de las alianzas con las estancias de la Universidad de Costa Rica, Instituto Costarricense del Deporte y la Recreación (INCODER) y Instituto Costarricense sobre Drogas de Costa Rica ( ICD) una vez concluida este proceso, se planificó las actividades que se desarrollaran durante el campamento.

En una segunda etapa, está representado por la ejecución del campamento, y se procede a trasladar a los estudiantes a un escenario fuera del aula y del campús 
universitario en un parque recreativo rodeado de bosque húmedo, en compañía de muchos elementos de la naturaleza: espacio físico amplio, zonas verdes extensas, ubicado en la provincia de Alajuela cantón de San Pedro de Poas. El campamento tuvo una duración de dos días y medio. Las técnicas de recolección de información que se utilizaron en los grupos de discusión fue a través de la planificación de un instrumento que es una agenda de trabajo donde se especificó cada actividad con un nombre ficticio, en grupos pequeños con un facilitador, se hace uso de dinámicas diversas con la finalidad que el y la estudiante expresen sus sentimientos de varias formas, luego el docente hace una observación participante, el facilitador se involucra en el proceso, utilizando una guía de observación (anexo 1), basado en esta misma metodología habilidades para la vida, donde se enfatizó las destrezas psicosociales del estudiante de Enfermería, tales como: pensamiento crítico, empatía, solución de problemas y conflictos, manejo de sentimientos y emociones, el docente registra la información a través de un diario de campo.

Otras actividades fueron desarrolladas a través de el leguaje del cuerpo, como fue la expresión corporal con el acompañamiento de la músico- terapia dirigido por un facilitador, y se utiliza el acompañamiento del entorno, los sonidos y elementos de la naturaleza tales como: noche, la luna, las estrellas, viento, árboles, lluvia, luz. Además también se planifica una actividad que se llama la noche de talentos donde los estudiantes expresan a nivel grupal sus capacidades en la música, poesía, el teatro en otras.
Algunas otras técnicas que acompañan este proceso metodológico son la estimulación de la inteligencia emocional y la programación neurolingüística. Las cuales son promovidas a través de la expresión de sentimientos con la pintura, cada participante reflexiona con los colores primarios y figuras o formas que más se identifique, al final la expresión se hace a nivel grupal donde los jóvenes explican cada boceto.

El fomento del ejercicio y el deporte se planifica con un rally con pistas a través del bosque, esta actividad se desarrolla en pequeño grupo para fortalecimiento de la creatividad, liderazgo, cooperación, solidaridad y la resolución de conflicto.

Al final de la actividad de campamento se realizó la evaluación de la misma, en forma escrita y participativa a través de preguntas generadoras promovida por el docente facilitador, el estudiante de enfermería opina sobre el campamento y las actividades desarrolladas. Para concluir, se hace un cierre pedagógico que se le ha denominado el círculo mágico, dirigidos por el equipo de docentes, cual es un ejercicio que hace el estudiante de Enfermería en forma individual manifestando sus sentimientos y es reforzado por el resto del grupo con el reconocimiento de su autoestima.

En la tercera etapa, los docentes participantes, sistematizaron la información obtenida a través del diario de campo y de la observación participativa; para el análisis y discusión de los resultados que permitieron la retroalimentación del proceso como actividad académica e investigativa.

\section{RESULTADOS.}

De la información recopilada durante el estudio, se realizó una sistematización de 
la misma, las cuales corresponden a cada una de las habilidades para la vida que propone Mantilla (2001), estos datos se exponen a continuación:

El conocimiento de sí mismo(a) se evidenció en las y los participantes del estudio a través de la verbalización de algunos comentarios, dentro de estos se encuentran los siguientes:

"..me hizo crecer como persona porque, me ayudo a conocerme mejor, de tal forma que así puedo entender la mejor manera en que me comporto y la forma cómo reaccionó ante diversas situaciones" (Estudiantes de Enfermería)

"Me redescubrí y me enfrente a mis temores y verdades más oscuras, pero gracias al apoyo de quienes estuvieron a mi lado logré vencer y abrazar nuevamente a mi vida..." (Estudiante de Enfermería)

"...constituye una experiencia que aporta grandes beneficios respecto al conocimiento personal, ya que se nos brinda las herramientas para que nosotros mismos seamos quienes exploremos nuestro yo interior" (Estudiante de Enfermería)

"Como la persona se encamina cada vez más hacia la auto aceptación, logrando encontrar una mejor percepción de sí mismo" (Estudiante de Enfermería)

"Gracias por ayudarme a crecer y por ver lo hermosa que es la sonrisa de los demás, por darme la bendición de llorar cuando se abraza a otra persona que en realidad soy yo misma"(Estudiante de Enfermería)

A continuación se citan algunos de los relatos que expresaron los y las participantes, los cuales corresponden a comportamientos que evidencian la habilidad para la vida de la empatía, así como también fue posible observar durante la actividad denominada "El Rally" conductas de acercamiento en las interacciones sociales tales como solidaridad, acompañamiento, entre otras.

"...se realizaron actividades que son muy oportunas $y$ me ayudan a sensibilizarme con la historia de vida y la realidades de los y las compañeros" (Estudiante de Enfermería)

"...el hecho de conocer más sobre otras personas que comúnmente me rodean pero debido a mi aislamiento no conozco bien." "La sensación de sentir el afecto $y$ aprecio de otras personas lo cual no ciento nunca porque las personas se alejan de mí o me causan daño" (Estudiante de Enfermería)

"Entre los músculos a doloridos, cansancio físico y la voz ronca...Pero esos músculos duelen de tanto abrazar a mis amigas de sostenerlos y de darles apoyo." (Estudiante de Enfermería)

"Llevo una generación conmigo en quienes puedo confiar cuando desfallezca o me sienta sin fuerzas..." (Estudiante de Enfermería)

"Una gran experiencia de convivencia en la que no sentí en ningún momento ser rechazada sino más bien aceptada igual que todos (as)..." (Estudiante de Enfermería)

La comunicación efectiva entre los y las participantes se evidenció, en primer lugar, en la observación durante la realización de las diferentes actividades, se evidenció en la actividad denominada "La Casa de Cristal: los y las participantes se organizaron de forma verbal y no verbal para resolver diferentes pruebas". En segundo lugar, a través de los comentarios por parte de los y las 
mismas, a continuación se enuncian algunos de estos.

"Sonrisas, miradas que expresan mucho y crearon pensamientos, que hoy se acomodan para transformarme $y$ seguir en crecimiento, muchos retos...gracias" (Estudiantes de Enfermería)

“...pude relacionarme con más personas así que más conocidos a quien saludar..." (Estudiante de Enfermería)

"Conocí y trate a personas a las que nunca les había hablado y eso se siente hermoso..." (Estudiante de Enfermería)

La presencia de Relaciones Interpersonales fue evidente a lo largo de distintas actividades, como en la preparación para "La Noche de Talentos" los y las estudiantes durante los espacios de tiempo para reuniones compartieron ideas, propuestas, comentarios, bromas, entre otras. Además, expresaron a través de comentarios dicha habilidad para la vida, algunos de estos son los siguientes.

"...el hecho de conocer más sobre otras personas que comúnmente me rodean pero debido a mi aislamiento no conozco bien...tuve la oportunidad de relacionarme más..." (Estudiante de Enfermería)

“...la sensación de sentir el afecto y aprecio de otras personas lo cual no siento nunca porque las personas se alejan de mí o me causan daño cambio, porque aquí me relacione con personas que cambiaron eso..." (Estudiante de Enfermería)

“...mayor unión con los que ya tenía, mayor confianza, me llevo mucha felicidad y paz en mí corazón..." (Estudiante de Enfermería)

“...conocí a muchas personas y entendí que no podemos juzgar a una persona por lo que ves sino sabes que hay en su corazón.. en reuniones. (Estudiante de Enfermería)

La habilidad para la toma de decisiones, se presentó frecuentemente durante las actividades, durante la distribución de funciones, como la de limpieza y orden de las instalaciones durante el campamento, los y las participantes organizadamente decidieron la distribución de tareas. También en diferentes comentarios se evidencia dicho comportamiento, como hacer cambios en su vida a partir de esta etapa de reflexión, a continuación algunos relatos:

"...mayor unión con los que ya tenía, mayor confianza, me llevo mucha felicidad y paz en mí corazón..." (Estudiante de Enfermería)

"Ganas de luchar, la convicción de que puedo, que es cuestión de actitud, voluntad y esmero..." (Estudiante de Enfermería)

“...ahora decidí que voy a ser mejor y ya se que soy bella y especial..." (Estudiante de Enfermería)

"Me volví a encontrar, Había creado una personalidad que no me pertenecía, ahora me di cuenta que soy Yo y que no debo porque cambiar nunca, Hay que ser uno mismo siempre..." (Estudiante de Enfermería)

“...me permitió pensar en mí estar conviviendo con mis problemas y tomar decisiones de suma importancia para mí "(Estudiante de Enfermería)

La solución de problemas o conflictos se observó durante varios espacios, en la clausura "La Devolución", varios y varias participantes concluyeron algunos eventos conflictivos que habían abierto en otros espacios, por ejemplo conflictos ocurridos durante módulos pasados de la carrera; en "La Casa de Cristal" fue 
necesario la solución de diferente tipo de conflictos dentro del grupo para poder concluir satisfactoriamente la actividad, igualmente el grupo solucionó el problema de la deserción de algunos y algunas participantes durante el juego. Mediante sus relatos en el cierre del campamento, se evidenció dicha habilidad para la vida, a continuación se mencionan algunos:

"Voy cansada pero muy feliz por la cada cosa que resolví, así como por las muchas cosas aprendidas" (Estudiante de Enfermería).

Con respecto al pensamiento crítico, éste se evidenció frecuentemente durante la reflexión personal de los y las estudiantes al evaluar los cambios percibidos a nivel personal durante el proceso, a continuación se citan algunos relatos.

"Me llevo gran alegría, entendimiento de lo necesario que es compartir con los demás, conocer más a mis compañeras y saber que puedo confiar en ellas. Fortaleza y confianza en mí misma" (Estudiante de Enfermería)

"Una perspectiva diferente del grupo y de mí misma..." (Estudiante de Enfermería)

"Me llevo un nuevo resurgir, una nueva yo, una mejor visión de vida, muchas amigas $y$ toneladas de voluntad..." (Estudiante de Enfermería)

".....me hizo crecer como persona, me ayudo a conocerme mejor, de tal forma que así puedo entender la mejor manera en que me comporto y la forma como reaccionó ante diversas situaciones" (Estudiantes de Enfermería)

"Se favoreció la unión de grupo y conocer más allá de lo académico a muchos, además de darse cuenta de la importancia que tiene para nosotros esas personas que nos rodean diariamente, así como nosotros con ellos "(Estudiante de Enfermería)

La habilidad para el manejo de emociones y sentimientos se manifestó en muchos momentos, a través de ejercicios que promovieron la inteligencia emocional y la programación neurolingüística. Algunos de los comentarios expresados por los y las participantes que evidencian dicha competencia son los siguientes:

"Me llevo a mi misma solamente que ésta vez alguien que no conocía una mujer segura, plena, confiada y muy feliz..." (Estudiante de Enfermería)

"Un mejor concepto de mí misma, descubrí que puedo vencer el miedo y ser muy útil para los demás. También que el trabajo en equipo es fundamental y que en mí hay una gran persona digna $y$ dichosa de estar en este mundo" (Estudiante de Enfermería)

"Muchas cosas, demasiadas, tengo muchos sentimientos encontrados, porque en este momento tengo muchas ganas de llorar y reír, estoy feliz y triste, feliz por la experiencia que he vivido $y$ triste porque ya termino, pero me llevo muchas cosas valiosas dentro de mí..."(Estudiante de Enfermería)

"Ganas de luchar, la convicción de que puedo, que es cuestión de actitud, voluntad y esmero..."(Estudiante de Enfermería)

".....me hizo crecer como persona, me ayudo a conocerme mejor, de tal forma que así puedo entender la mejor manera en que me comporto y la forma como reaccionó ante diversas situaciones" (Estudiantes de Enfermería)

"Gracias por ayudarme a crecer y por ver lo hermosa que es la sonrisa de los demás, por darme la bendición de llorar cuando se abraza a otra 
persona que en realidad soy yo misma“(Estudiante de Enfermería)

Por medio de comentarios y conductas observadas durante el proceso, se identificaron conductas propias y características de la programación neurolinguistica, entre estas la identidad por medio del conocimiento de si mismo(a), el medio y el entorno a través del uso adecuado de los diferentes espacios en cada una de las actividades, la presencia de diferentes capacidades representadas en la toma de decisiones y juicio crítico, así como en el de manejo de tensiones y estresores.

El conocimiento de sí mismo(a) se evidenció en las y los participantes del estudio a través de la verbalización de algunos comentarios, dentro de estos se encuentran los siguientes ejemplos:

".....me hizo crecer como persona, me ayudo a conocerme mejor, de tal forma que así puedo entender la mejor manera en que me comporto y la forma como reaccionó ante diversas situaciones" (Estudiantes de Enfermería)

"Me redescubrí y me enfrente a mis temores y verdades más oscuras, pero gracias al apoyo de quienes estuvieron a mi lado logré vencer y abrazar nuevamente a mi vida..." (Estudiante de Enfermería)

"...constituye una experiencia que aporta grandes beneficios respecto al conocimiento personal, ya que se nos brinda las herramientas para que nosotros mismos seamos quienes exploremos nuestro yo interior" (Estudiante de Enfermería)

"La persona se encamina cada vez más hacia la auto aceptación, logrando encontrar una mejor percepción de sí mismo" (Estudiante de Enfermería)

\begin{abstract}
"Gracias por ayudarme a crecer $\mathbf{y}$ por ver lo hermosa que es la sonrisa de los demás, por darme la bendición de llorar cuando se abraza a otra persona que en realidad soy yo misma“(Estudiante de Enfermería)
\end{abstract}

La estrecha relación entre el espacio, el entorno, y las actividades fue posible determinarlas a través del manejo de factores limitantes y/o potencializadores, como lo fueron el clima, la oscuridad, el barro, las alturas, la lluvia incesante, entre otros, ya que los y las estudiantes fueron capaces de participar activamente en el proceso, y manejar positivamente los sentimientos y emociones asociados a esos factores.

\section{DISCUSIÓN.}

El proceso de investigación participativa en el que se desarrolló el campamento permitió, interactuar en forma activa con los sujetos de la investigación que estuvo caracterizado por los estudiantes de enfermería, además de combinar la docencia en el trabajo educativo y la acción en las actividades que se ejecutaron. Como contribución les aporto a los y las estudiantes, el fortalecimiento del desarrollo personal, profesional y dentro de este, la planificación de una estrategia educativa en la modalidad de campamento para el abordaje de la población de adolescentes y jóvenes.

A partir del fortalecimiento personal del estudiante de enfermería, como un proceso sistemático, se trabajo con la metodología de las habilidades para la vida, que el autor Mantilla (2000) delimita como un grupo genérico de habilidades o destrezas psicosociales que les facilitan a las personas enfrentar con éxito las exigencias y desafíos de la vida diaria. Por lo tanto, podemos entenderlas 
en los jóvenes como el descubrimiento de las habilidades para conducirse de cierta manera, de acuerdo con la motivación individual y el campo de acción que tenga la persona, dentro de sus limitaciones sociales y culturales, y en este aspecto es donde estas habilidades psicosociales es relacionan con los factores de protección y en especial con la resiliencia que fue manifestado por los mismos estudiantes en sus relatos. También se obtuvo un avance en la cohesión de grupo de la generación del tercer año de la carrera, ya que las mismas acciones tendieron al propósito del trabajo de equipo. Otras fortalezas descubiertas a nivel individual y también narradas por los jóvenes fueron: la confianza en sí mismos, mejoraron las relaciones interpersonales, lograron identificar sus capacidades, alcanzaron la motivación en la convivencia, además exteriorizar su propia creatividad en las actividades ejecutadas, otras experiencias descritas por los estudiantes fueron: un fortalecimiento de la autoestima, la utilización de la comunicación efectiva, el descubrimiento de fortalezas y debilidades, la reflexión acerca de cómo revolver las situaciones críticas y emocionales, el reconocimiento de su propia historia de vida, que estas según Mantilla(2000) son herramientas importantes en el manejo las distintas situaciones cotidianas como también de riesgo social.

En el plano profesional el estudiante de enfermería durante el avance de las actividades del campamento, le permitió aprender sobre la enseñanza de estas habilidades, que requiere de una aproximación metodológica diferente en el abordaje de programas dirigidos a la población de adolescentes y jóvenes, desde una visión, como agente facilitador $\mathrm{y}$ propiciador del el proceso de aprendizaje.

Dentro de la capacidad psicosocial de los jóvenes estudiantes de enfermería se exploro la inteligencia emocional, la cual se centro exclusivamente en el procesamiento emocional de la información y en el estudio de las capacidades de los jóvenes.

De esta manera otros autores también visualizan el enfoque de la inteligencia emocional como habilidades que están intrínsicos en las personas y que deben explorarse según Fernández:

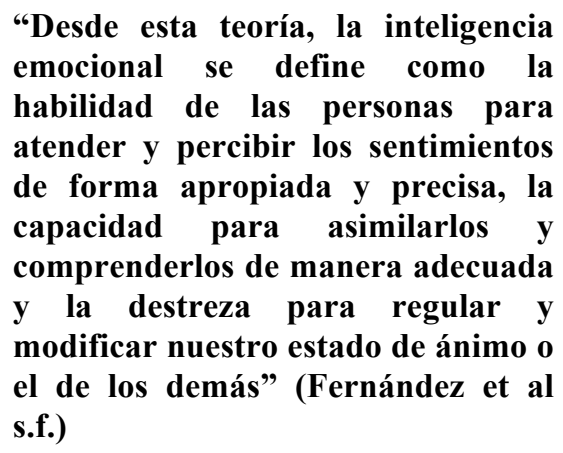

Durante el proceso continuo de las actividades de ejecución del campamento, el estudiante de enfermería reconoce desde su propia perspectiva sus habilidades, situación que propicia un factor protector, positivo $y$ de empoderamiento en la persona joven.

Otra estrategia desarrollada en este proceso, fue el mecanismo de ayuda en el aprendizaje que formula según Dilts (2004) fue la programación neurolingüística (P.N.L) como un modelo de la forma en que las personas estructuran sus experiencias individuales en la vida. El propósito de la programación neurolingüística es ser útil, incrementar las opciones y mejorar la calidad de vida. Algunos de los 
estudiantes de enfermería mencionaron a través de las diversas actividades que se desarrollan la expresión de sentimientos y emociones a través de la pintura, evidenciaron situaciones conflictivas $\mathrm{y}$ dolorosas por las que ha atravesado en su vida y fueron compartidas grupalmente.

De acuerdo con Peralta (2002) las situaciones de beneficio del crecimiento individual como el ayudar a las personas a acrecentar su autoestima puede inducir situaciones beneficiosas y reforzar así los recursos para la vida futura. Para los jóvenes estudiantes, esa auténtica necesidad de fraguar su identidad y sentirse bien consigo mismo, es un paso para seguir adelante y estar preparado para asumir las responsabilidades subsiguientes.

Zambrano citada por Gessen y Gessen (2002) propone algunas estrategias básicas en la programación neurolingüística, las cuales se denominan submodalidades que son variantes de los modelos de los sistemas de representación y expresan las distintas maneras como nuestro cerebro clasifica y codifica la experiencia.

Existen tres tipos de submodalidades, las Visuales como color, distancia, profundidad, claridad, contraste y luminosidad. Las Auditivas como volumen, tono, ritmo $\mathrm{y}$ pausas, $\mathrm{y}$ Kinestésicas como temperatura, vibración, textura, presión, movimiento y peso. Aplicando actividades como los juegos la combinación de estas técnicas es posible emplear correctamente en un modelo de programación neurolingüística, ya que como Andreu y García (s.a.) señalan que desde siempre se ha considerado el juego como un elemento intrínseco de la personalidad humana, potenciador del aprendizaje la práctica del ejercicio y del deporte. De manera que el juego forma parte de esta etapa en el campamento y le permite a su vez al estudiante de enfermería el manejo de su corporalidad, a través del lenguaje del cuerpo que se promueve en el grupo con el acompañamiento de la musicoterapia para conducir la expresión corporal, que a su vez favorece según manifestaron los jóvenes el alivio del stress.

Todo lo anterior permite concluir que no existe una realidad única, sino realidades personales, unidas por la cultura, los estudiantes de enfermería evidencian estas realidades en sus expresiones, ya que responden a situaciones diferentes de comportamiento de conducta de maneras de actuar y de pensar. Asimismo representan la clave para que los jóvenes reflexionen sobre los valores, las emociones y formas de expresión que también pueden cambiar meditando sobre "la percepción de la realidad" y de la auto- imagen de las personas, situación que ha sido vivenciada por los estudiantes de enfermería con la participación en este proceso de aprendizaje y de cambio.

\section{CONCLUSIONES.}

Al finalizar esta etapa de discusión análisis es posible exponer las siguientes conclusiones:

- El campamento se presenta en el curso del módulo de intervención de enfermería en la adolescencia como parte del programa de Licenciatura de la Universidad de Costa Rica, se enseña como una aproximación metodológica diferente, a través de esta modalidad, dirigido a los estudiantes de enfermería, como una propuesta educativa para la promoción del desarrollo humano y la 
prevención de los problemas psicosociales de los jóvenes del mundo contemporáneo.

- La metodología de abordaje sobre las habilidades para la vida, es una estrategia que ha brindado nuevas experiencias en el campamento, que han fortalecido la capacidad de los estudiantes de enfermería para enfrentar a las dificultades de la vida cotidiana, pero que además permitió el fomento y la estimulación de el desarrollo de los valores y las fortalezas de sí mismos.

- $\quad$ Esta propuesta educativa a través del plan de estudios de la licenciatura en enfermería, constituida en el modulo de la adolescencia, busca formar a personas con más criticidad, solidaridad, autonomía, respeto y tolerancia pero específicamente ha permitido establecer la conciencia de la realidad del mundo que los rodea y a su vez permite la reflexión acerca de su proyecto de vida como persona joven.

- Esta metodología de abordaje en la modalidad de campamento le permite al estudiante de enfermería obtener el conocimiento en el diseño de programas educativos dirigidos a la niñez y adolescencia además de facilitarle el proceso de interacción con esta población.

- $\quad$ En el equipo de docentes que participan planificando y ejecutando esta actividad de campamento, con la aplicación de distintas metodologías utilizadas como las habilidades para la vida, la inteligencia emocional y la programación neurolinguista, le han permitido a este grupo académico visualizar al estudiantado como personas humanas que a través del desarrollo de su vida requieren un espacio fuera del aula para hacer un reconocimiento de su persona a nivel individual, ya que son jóvenes algunos adolecentes por su edad o por el mismo proceso de maduración desde su crecimiento y desarrollo, que atraviesan obstáculos, fracasan, tienen dificultades, alegrías, tristezas y metas por cumplir que necesitan ser escuchadas y fortalecidas.

\section{BIBLIOGRAFÍA.}

Albala, C., Salinas, J., Vio, F. (2004). Bases, prioridades y desafíos de la promoción de la salud. Santiago: Universidad de Chile . Rev. chil. nutr. [online]. 2004, vol. 31, supl. 1 [citado 200906-14], pp. 183-199. Disponible en: < http://www.scielo.cl/scielo.php. Tomado el $12 \mathrm{de}$ junio del 2009.

Andreu, M. García, M. (s/f) Actividades lúdicas en la enseñanza de LFE: el juego didáctico. Memorias del Primer Congreso Internacional de Español para fines específicos. Valencia: Universidad Politécnica.

Badilla, H. (1999). Para comprender el concepto de resiliencia. Revista costarricense de trabajo social. Mayo 1999. (9) p. 22-29.

Cooper, R., Sawaf, A. (1998). La Inteligencia Emocional aplicada al liderazgo y a las organizaciones. Barcelona: Editorial Norma.

Dilts. Roberts. (2004) Coaching, herramientas para el cambio. Madrid: Ediciones Urano.

Fernández, et al. (s/f) Cultura, inteligencia emocional percibida y ajuste emocional: un estudio preliminar. Revista REME. (4)8-9

Gessen, M. Gessen, V. (2002.) Programación Neurolinguíística. EDUCERE, TRASVASE, 6(19), 341.

Goldam, H. (1999). Psiquiatría General. México D. F.: Editorial El Manual Moderno S.A.

Grinder, A. et al (s/f). No hay consejos sólo sugerencias. Material para los cursos del Instituto Internacional de Programación Neurolingüística e Inteligencia Emocional (I.I.P.N.L). Santiago. 
Mantilla, L. (2000). Habilidades para vivir. $2^{\text {da }}$ Edición. Política Nacional de prevención, detección y atención de la violencia intrafamiliar. Gobierno de Bogota.

Martínez, Miguel. (2006). La investigación cualitativa. Síntesis conceptual. Revista Facultadad de Psicología, vol. $9 \mathrm{~N}^{\circ} 1$, pp. 123-146, Venezuela.
Peralta, P. et al. (2002). Proyecto para aplicar técnicas de P.N.L. Revista Pharos (9)1, 130-148. Chile.

Vejarano, M. (1983) La investigación participativa en América Latina. México. D.F.: CREFAL (Centro Regional de Educación de adultos y alfabetización funcional para América Latina.

\section{ANEXO 1}

\section{Habilidades para Vivir y la convivencia pacífica}

\begin{tabular}{|c|c|}
\hline Destreza psicosocial & Le permite al individuo... \\
\hline Pensamiento crítico & $\begin{array}{l}\text { - Darse cuenta que la realidad puede interpretarse desde ángulos y } \\
\text { perspectivas diferentes. } \\
\text { - Comprender que existen puntos de vista diferentes sobre un } \\
\text { mismo tema o asunto. } \\
\text { - Tomar distancia de los propios pensamientos, emociones, } \\
\text { perjuicios, valores y opiniones para comprenderlos y manejarlos. }\end{array}$ \\
\hline Empatía & $\begin{array}{l}\text { - "Ponerse en los zapatos del otro" y comprender mejor sus } \\
\text { puntos de vista, emociones y reacciones. } \\
\text { - Comprender que la diferencia es intrínseca a la existencia } \\
\text { humana y un factor de crecimiento personal y social, y ser más tolerante } \\
\text { fon los demás. } \\
\text { - Comportarse en forma menos agresiva. }\end{array}$ \\
\hline $\begin{array}{c}\text { Solución de problemas } \\
\text { y conflictos }\end{array}$ & $\begin{array}{l}\text { - Identificar problemas, sus causas y alternativas de solución. } \\
\text { - Comprender que las situaciones y relaciones de conflicto son } \\
\text { procesos, y representan momentos de oportunidades y soluciones } \\
\text { productivas. } \\
\text { - Aprender a negociar la solución de conflictos. }\end{array}$ \\
\hline $\begin{array}{c}\text { Manejo de sentimientos } \\
\text { y emociones }\end{array}$ & \begin{tabular}{|ll} 
- & Aprender que la ira es una emoción humana y normal. \\
a ira. & Aprender a reconocer los cambios fisiológicos que acompañan \\
- & Reconocer que la ira puede usarse en forma constructiva. \\
- & Aprender y poner en práctica estrategias para expresar la ira en \\
orma no destructiva.
\end{tabular} \\
\hline
\end{tabular}


